\title{
The Dip-Slide:
}

\section{a modified dip-inoculum transport medium for the laboratory diagnosis of infections of the urinary tract}

\author{
By G. R. E. NAYLOR \\ Public Health Laboratory Service, Cambridge \\ AND DENNIS GUTTMANN \\ Addenbrooke's Hospital, Cambridge
}

(Received 22 March 1967)

Determination of the concentration of bacteria in freshly voided urine can assist in the diagnosis of urinary tract infections, particularly by helping in the distinction between growth on culture which reflects infection and that which is the result of contamination during collection (Kass, 1956). Mackey \& Sandys (1965, 1966) described a dip-inoculum transport medium which enabled such quantitative determinations to be made when rapid access to a laboratory is a problem, thus avoiding misleading impressions produced by the proliferation of organisms in urine after collection and before arrival at the laboratory. This paper describes a dip-slide which is based on Mackey and Sandys's idea of a solid transport medium inoculated by dipping and is an alternative to their nutrient medium filled spoon. The advantages of the dip-slide are that it is readily made from easily available materials, it is cheap, two different media can be inoculated simultaneously if desired, an area of 2 sq. in. of each medium is inoculated and colony counts are easy. Briefly, the dip-slide consists of a $3 \times 1$ in. microscope slide coated on one or both sides with nutrient medium for a $2 \mathrm{in}$. length; the slide is housed in a cylindrical aluminium container which grips it firmly with the uncoated portion of the slide at the capped end. In use, the slide is held by the uncoated portion, dipped in fresh urine and replaced in the container. On arrival at the laboratory, and a delay is of no consequence, the unopened container is incubated and the slide examined the following day. The number of colonies on the slide is a measure of the bacterial concentration in the fresh urine.

\section{Preparation of dip-slides}

\section{METHODS}

Microscope slides, $3 \times 1$ in., are sterilized in bulk. A suitable rack to hold the slides is described but the method could easily be varied (see Plate 1). A row of nine slides is clipped with $1 \frac{1}{4} \mathrm{in}$. spring clips ('Bulldog' brand, M. Myers and Son Ltd.) to a $20 \times 2 \mathrm{~mm}$. Duralumin bar about $15 \mathrm{in}$. long fixed (with Evo-Stik) to large rubber bungs. The slides are handled with sterile forceps but the bar and clips are not sterile. Using a $10 \mathrm{ml}$. pipette, $1 \cdot 5-2 \mathrm{ml}$. of molten agar medium at $50-55^{\circ} \mathrm{C}$. is run on to each slide where it forms a layer over a 2 in. length of the slide. When 
the medium has set, in 2 or $3 \mathrm{~min}$., the whole bar with attached slides is turned over and a second medium run on to the other side of the slides. MacConkey's agar and nutrient agar are a suitable pair of media; this combination allows the growth of both Gram-negative rods and Gram-positive cocci which may cause urinary tract infections, proteus strains do not swarm on the MacConkey's agar and both media are commonly used for other purposes so that a special medium does not have to be prepared. But other media could be used.

Extruded aluminium screw-capped containers $27 \times 75 \mathrm{~mm}$. (The Metal Box Company Ltd.), commonly used in the Public Health Laboratory Service as faeces containers, are sterilized without a cardboard liner in the cap but with a $1 \mathrm{in.} \mathrm{disk} \mathrm{of} \mathrm{thin} \mathrm{white} \mathrm{filter} \mathrm{paper} \mathrm{in} \mathrm{the} \mathrm{bottom;} \mathrm{these} \mathrm{disks} \mathrm{are} \mathrm{cut} \mathrm{on} \mathrm{a} \mathrm{metal}$ punching machine, many thicknesses at a time, but 0.7 in. squares could be used instead. The slides are put into the aluminium containers by slipping the container over the coated slide and unclipping the slide at the same time. A slight squeeze of the aluminium container makes this easier. As the container is brought upright the slide drops into the container and rests on the disk of filter paper at the bottom. The slide is not handled. The cap of the container is sealed with two $2 \mathrm{in}$. lengths of insulating tape (Rotunda P.V.C. adhesive tape $\frac{5}{8}$ in., Rotunda Ltd., Denton, Manchester). The slides are prepared under a half cylindrical dome of Perspex, hinged in two halves, attached to a baseboard $17 \times 13$ in., intended for the display of cheese (Code Number C.B. 1, P. L. Display Co. Ltd., Southdown Works, Kingston Road, Raynes Park, London, S.W. 20).

The slides can be stored, preferably at $4^{\circ} \mathrm{C}$., for a month or more: slides used after storage for a year at $4^{\circ} \mathrm{C}$. have given the same results as freshly prepared slides. Difficulty with stored slides has arisen in the past from the growth of airborne mould spores contaminating the nutrient agar medium during preparation of the slides; because the container is opaque the contamination is not seen until the slide is removed for use. If the slides are carefully prepared only an occasional slide is contaminated, but the difficulty can be avoided by adding cycloheximide (Acti-dione, The Upjohn Company) to the nutrient agar medium at a concentration of $100 \mu \mathrm{g} . / \mathrm{ml}$. This was found to inhibit the growth of moulds on medium deliberately exposed to airborne contaminants, and such a concentration did not interfere with the growth of bacteria, including nine strains of coagulase negative staphylococci, isolated from pyogenic infections of the urinary tract, the number and size of the colonies being unchanged. We have found that the addition of cycloheximide to nutrient agar does not alter the relationship between dip-slide colony counts and pour plate viable counts (Guttmann \& Naylor, 1967).

\section{Method of use}

The plastic tape sealing the container is peeled off and stuck down the side of the container for labelling. The slide is held in the fingers by the uncoated portion and dipped in the urine to immerse the agar medium. The slide is then drained by touching a corner to absorbent paper, which need not be sterile. The slide is replaced in the container, squeezing the container slightly if necessary, and the cap screwed on lightly; it is not resealed. The container should be left standing 
upright for a few minutes so that any more urine which drains off the slide can be absorbed by the filter paper disk in the bottom of the container.

After delivery at the laboratory, the container is incubated overnight. The number of colonies on the slide can be counted, and the purity or otherwise of the growth assessed. Subcultures can be taken if necessary. The slide can be conveniently held in a pair of Spencer Wells forceps with short lengths of fine rubber tubing on the jaws. The slide should be discarded into disinfectant; if it is put back in the container, the lip and hence the rim of the cap may be contaminated by bacterial growth scraped off the slide.

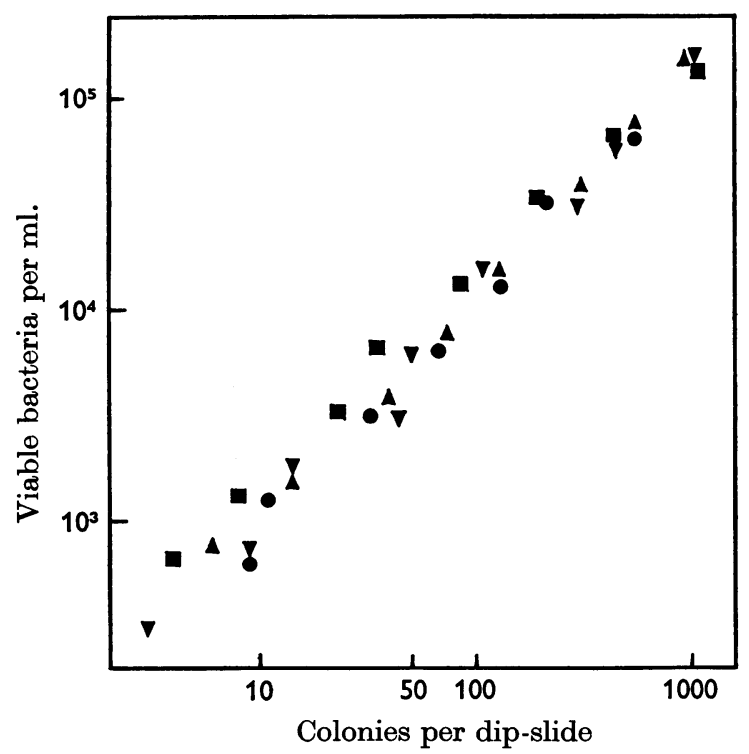

Fig. 1. Relationship between number of colonies on the dip-slide and the viable bacterial count of the fluid in which the slide has been dipped. - Escherichia coli, - Proteus mirabilis (dip-slide counts and plate counts on MacConkey's agar). $\Delta$ Faecal streptococcus, $\nabla$ coagulase negative staphylococcus (dip-slide counts on nutrient agar and plate counts on blood agar).

\section{RESULTS}

Figure 1 shows the relationship between colony counts on dip-slides and viable bacterial counts of the fluid in which the slide is dipped. Overnight broth cultures of organisms from pyogenic infections of the urinary tract were diluted in quarterstrength Ringer's solution. Viable bacterial counts were determined by surface colony plate counts. All dip-slide and plate counts are the average of at least three determinations. The number of colonies on the dip-slide is directly proportional to the viable bacterial count of the fluid in which the slide has been dipped. Between 50 and 100 colonies on an area of 2 sq. in. on the dip-slide corresponds with a viable bacterial count of $10^{4}$ per $\mathrm{ml}$.

Colonies on the dip-slide are easy to count. Colony counts below 20 are unlikely to be reliable because of sampling variation; counts approaching and above 500 are increasingly difficult and inaccurate because of overcrowding of colonies; 
above 1000 colonies the growth becomes semi-confluent. Therefore the dip-slide colony count is most reliable and convenient in the range from 20 to 500 .

In general, contaminant bacteria in fresh urine are fewer than $10^{4}$ per $\mathrm{ml}$. whereas infecting organisms exceed $10^{5}$ per $\mathrm{ml}$. (Kass, 1956). Therefore the differentiation of viable bacterial counts below $10^{4}$ or above $10^{5}$ is of less importance in distinguishing between contamination and infection.

We have compared dip-slide colony counts with viable bacterial counts by the pour plate method on 385 samples of urine from patients attending a urinary infection clinic (Guttmann \& Naylor, 1967). We found that fewer than 40 colonies on the dip-slide always corresponds with a pour plate viable count of less than $10^{4}$ organisms per ml., and that viable counts greater than $10^{5}$ organisms per ml. always correspond with more than 200 colonies on the dip-slide; heavily infected specimens containing more than $10^{6}$ organisms per ml. give semi-confluent or confluent growths on the dip-slide. The dip-slide thus provides its maximum discrimination over the range of viable bacterial counts which is most helpful in the diagnosis of urinary tract infections.

After a little experience, dip-slides can be used to estimate the numbers of viable bacteria in urine as significant, not significant or doubtful, without counting colonies. As the area of inoculated medium is 2 sq. in., features of colonies can be observed even when the urine contains many thousand organisms per $\mathrm{ml}$.; consequently mixed growth can be detected as an additional indication of contamination.

\section{Uses of dip-slides.}

The dip-slide provides a measure of the bacterial concentration in freshly voided urine as supplementary information to that obtained by microscopy and conventional cultural methods including sensitivity tests. Dip-slides overcome difficulties in laboratory diagnosis caused by the proliferation of contaminating bacteria in urine after collection and consequently they are of value in the diagnosis of urinary tract infections in general practice where the laboratory is some distance away. Their main use is when urine specimens have to be sent to the laboratory by post, but they are also useful in evening surgeries. They are particularly helpful when there is any problem in diagnosis. They have been in use for a year without difficulty. They are unnecessary when urine specimens can be delivered quickly to the laboratory.

Dip-slides are also useful in screening for symptomless bacteriuria. When the majority of those to be examined are likely to be free from infection, culture of the urine on a dip-slide can be used as the sole test. In such circumstances, the ease of inoculating dip-slides and reading the results more than offsets the need to collect follow-up specimens for full examination from anyone giving a dip-slide result in the significant or doubtful range.

Dip-slides could also be useful in investigations in which it was desirable to follow the approximate bacterial concentration in urine at frequent intervals during the day and night. Dip-slides could be inoculated at intervals by nursing staff, and incubated and read in batches. 
Journal of Hygiene, Vol. 65, No. 3

Plate 1

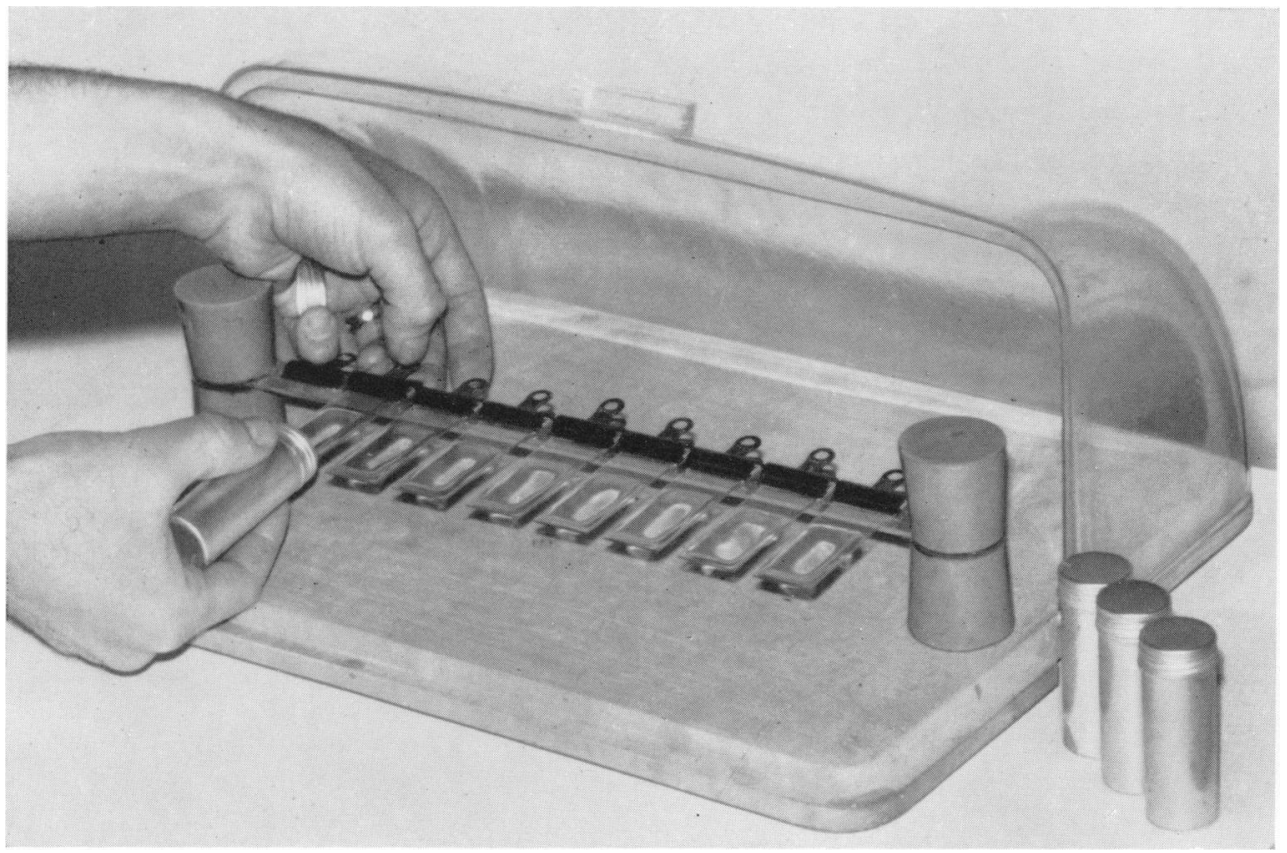




\section{SUMMARY}

The dip-slide, consisting of a glass microscope slide coated with nutrient medium and inoculated by dipping in freshly voided urine, provides a simple measure of the bacterial concentration in fresh urine. This is a useful supplement to the usual microscopy and culture of urine in the diagnosis of urinary tract infections in general practice, when specimens cannot be delivered to a laboratory within several hours of collection, particularly when urine specimens have to travel by post. Dip-slides are also useful as the sole test in screening for symptomless bacteriuria.

We are grateful to the technical staff of the Public Health Laboratory, Cambridge, for their help in the method of preparation of the dip-slides and to the Medical Practitioners who have used them and given us their comments. We thank Mr L. F. H. Beard and Mr S. W. Patman for the photographs.

\section{REFERENCES}

Guttmann, D. \& Naylor, G. R. E. (1967). The Dip-Slide: an aid to quantitative urine culture in general practice. $B r$. med. J. iii, 343.

Kass, E. H. (1956). Asymptomatic infections of the urinary tract. Trans. Ass. Am. Physns. 69, 56.

MACKEY, J. P. \& SANDYs, G. H. (1965). Laboratory diagnosis of infections of the urinary tract in general practice by means of a dip-inoculum transport medium. Br. med. J. ii, 1286 .

MaCkey, J. P. \& SANDYs, G. H. (1966). Diagnosis of urinary infections (correspondence). Br. med.J. i, 1173.

\section{EXPLANATION OF PLATE}

Rack for preparation of dip-slides. The slides have been coated with nutrient medium and an aluminium container is being slipped over a slide. 